\title{
Verificación urgente de despidos intempestivos por parte de los Inspectores
} de Trabajo en Ecuador

\section{Urgent verification of untimely dismissals by Labor Inspectors in Ecuador}

\author{
Leonardo Toapanta-Jiménez \\ us.leonardotoapanta@uniandes.edu.ec \\ Universidad Regional Autónoma de los Andes, Santo Domingo \\ Ecuador \\ https://orcid.org/0000-0002-9489-8637 \\ Verónica-Susana Chicaiza-Chitupanta \\ verosu21@hotmail.com \\ Universidad Regional Autónoma de los Andes, Santo Domingo \\ Ecuador \\ https://orcid.org/0000-0002-9641-582X
}

Recepción: 15 de septiembre 2021

Revisado: 25 octubre 2021

Aprobación: 15 de noviembre 2021

Publicación: 01 de diciembre 2021 


\title{
RESUMEN
}

La presente investigación, de tipo documental se planteó como objetivo general, analizar jurídicamente la verificación urgente de despidos intempestivos por parte de los Inspectores de Trabajo en Ecuador. Desde el paradigma cuantitativo, se manejó para este tipo de estudio, documentos, textos legales, investigaciones científicas, sobre el tema tratado. Se empleó la técnica de la encuesta, a fin de obtener los resultados del proceso metodológico, y se aplicó a los abogados en libre ejercicio en materia laboral, tomando como población el registro del Foro de Abogados de Santo Domingo de los Tsáchilas, donde se determinó aleatoriamente una muestra de 65 abogados. Para la aplicación de esta encuesta, se utilizó Google Forms. Se evidenció la necesidad por la que atraviesa la Inspectoría del Trabajo de Santo Domingo, ya que como institución se ve limitada no solo en recursos económicos sino, en recursos humanos para prestar un pronto y eficaz auxilio al trabajador.

Descriptores: Derecho laboral; trabajador; derechos humanos; administración pública; discriminación. (Palabras tomadas de Tesauro UNESCO).

\begin{abstract}
The present investigation, of a documentary type, was proposed as a general objective, to legally analyze the urgent verification of untimely dismissals by Labor Inspectors in Ecuador. From the quantitative paradigm, documents, legal texts, scientific research were used for this type of study, on the subject matter. The survey technique was used, in order to obtain the results of the methodological process, and it was applied to lawyers in free exercise in labor matters, taking as population the registry of the Santo Domingo de los Tsáchilas Lawyers Forum, where it was determined a random sample of 65 attorneys. For the application of this survey, Google Forms was used. The need for the Santo Domingo Labor Inspectorate was evidenced, since as an institution it is limited not only in economic resources but also in human resources to provide prompt and effective assistance to the worker.
\end{abstract}

Descriptors: Labor law; worker; human rights; public administration; discrimination. (words takenfrom UNESCO Thesaurus). 


\section{INTRODUCCIÓN}

Analizar la realidad sobre la vulnerabilidad de los trabajadores frente a los empleadores con relación a los despidos intempestivos, es enfrentarnos a una realidad actual cada vez más marcada en el ámbito laboral, donde prevalece la superioridad del empleador frente al trabajador quien por su condición padecerá de injusticia e inequidad. Al trabajador, no solo se le presenta dificultades en el desarrollo de sus actividades o con relación a su remuneración o con los horarios de trabajo, sino que también carga con el riesgo de la inestabilidad o continuidad laboral ya que en cualquier momento puede ser objeto de un despido intempestivo. En este sentido, Dentro de la concepción clásica de política, el Derecho Laboral es definitivamente una herramienta para su implementación, puesto que su objetivo final es regular las relaciones para mejorar la calidad de vida de los actores. (Chiriboga Izquieta, Jiménez Franco y Toscanini Sequeira, 2018, p.227).

Frente a esta situación resulta imprescindible dotar a los trabajadores de mecanismos o medios eficaces que les permita hacer valer sus derechos enmarcados en el ámbito legal, por cuanto al existir una institución exclusiva que tiene como finalidad el amparo de los derechos del trabajador, en este caso la Inspectoría del Trabajo por intermedio de sus funcionarios quienes tienen la obligación de aplicar todos los medios necesarios que garanticen los derechos de los trabajadores, siendo estos los encargados de protegerlos de todo abuso de poder por parte de los empleadores. Por lo tanto, de acuerdo a Mundlak (2007), citado por Patlán Pérez, (2016, p.122) existen tres componentes del derecho al trabajo:

a) El derecho al trabajo como libertad (la libertad de ejercer una profesión) y no como esclavitud, sin dejar de mencionar la abolición del trabajo forzoso.

b) El derecho a tener trabajo, con la cuestionable obligatoriedad del Estado y los empleadores para proveer trabajo a las personas.

c) El derecho a tener un trabajo digno, en cuanto a condiciones y trato digno.

Sin embargo, cada vez es más común en la Inspectoría de Trabajo las audiencias por despidos intempestivos donde los trabajadores reclaman protección a sus derechos vulnerados por parte de sus empleadores, particularmente a la cancelación de la 
compensación por destitución intempestivo y otros rubros laborales, donde buscan llegar a acuerdos ante la difícil situación del trabajador. Para González Mendoza (2014), se vulnera el derecho del trabajador por cuanto:

La ilegalidad de esta forma de despido llamado intempestivo o ilegal es por no explicar causal alguna, pero, a diferencia del despido tempestivo, en su efecto el ilegal exige la paga de una liquidación o indemnización al trabajador lo que no ocurre en el supuesto despido legal. (p.21)

En ese contexto mediante las leyes y órganos de justicia se busca la protección de los trabajadores, al respecto se muestra en los siguientes cuerpos legales el interés del Estado en amparo y evitar la vulneración de los derechos

\section{Cuadro 1.}

Legislación.

\begin{tabular}{|c|c|}
\hline Normativa & Contenido \\
\hline Constitución de la República del Ecuador (2008) & $\begin{array}{l}\text { Artículo. 33.- El trabajo es un derecho y un deber } \\
\text { social, y un derecho económico, fuente de } \\
\text { realización personal y base de la economía. El } \\
\text { Estado garantizará a las personas trabajadoras } \\
\text { el pleno respeto a su dignidad, una vida } \\
\text { decorosa, remuneraciones y retribuciones justas } \\
\text { y el desempeño de un trabajo saludable y } \\
\text { libremente escogido o aceptado. } \\
\text { Artículo. 326.- El derecho al trabajo se sustenta } \\
\text { en los siguientes principios: } \\
\text { 1. El Estado impulsará el pleno empleo y la } \\
\text { eliminación del subempleo y del desempleo. } \\
\text { 2. Los derechos laborales son irrenunciables e } \\
\text { intangibles. Será nula toda estipulación en } \\
\text { contrario. }\end{array}$ \\
\hline Código del Trabajo (2005) & $\begin{array}{l}\text { Artículo. 5.- Protección judicial y administrativa. - } \\
\text { Los funcionarios judiciales y administrativos } \\
\text { están obligados a prestar a los trabajadores } \\
\text { oportuna y debida protección para la garantía y } \\
\text { eficacia de sus derechos. } \\
\text { Artículo. 542.- Atribuciones de las Direcciones } \\
\text { Regionales del trabajo. - Además de lo } \\
\text { expresado en los artículos anteriores, a las } \\
\text { Direcciones Regionales del Trabajo, les } \\
\text { corresponde: } \\
5 . \text { Visitar fábricas, talleres, establecimientos, } \\
\text { construcciones de locales destinados al trabajo y } \\
\text { a viviendas de trabajadores, siempre que lo } \\
\text { estimaren conveniente o cuando las empresas o } \\
\text { trabajadores lo soliciten; }\end{array}$ \\
\hline
\end{tabular}




\begin{tabular}{|l|l|}
\hline & Artículo. 545.- Atribuciones de los inspectores del \\
& trabajo. - Son atribuciones de los inspectores del \\
& trabajo: \\
& 3. Efectuar las visitas a las que se refiere el \\
numeral 5 del artículo 542 de este Código;
\end{tabular}

Elaboración: Los autores.

Es decir, de la normativa constitucional citada nos da un marco legal en donde podemos resaltar la naturaleza jurídica del trabajo al ser catalogado como un derecho el cual al tener este reconocimiento, se sustenta en los principios de irrenunciabilidad e intangibilidad, pero sobre todo el Estado se denomina protector del respeto a la dignidad de los trabajadores, lo cual significa una enorme responsabilidad para el Estado, ya que al hablar de dignidad de los trabajadores es un tema muy amplio en sentido de garantías a la cual el Estado está comprometido, puesto que no solo abarca un nivel individual sino colectivo, pero sobre todo es un derecho fundamental ya que de este se derivan otros derechos humanos que garantizan la vida digna no solo del trabajador, sino de su familia y por ende una colectividad estable.

De lo enunciado se colige que la dignidad humana es un valor propio e innato del hombre dotado de cualidades que lo hacen único y que lógicamente esta dignidad nace con el hombre, por lo tanto, tiene una gran trascendencia y el Estado a fin de garantizar el respeto al decoro humana del trabajador, debe agotar todos los medios necesarios para que se respete los derechos del trabajador a través de los organismos encargados de la vigilancia y control del sector laboral.

En la actualidad en el Ecuador, la entidad rectora de las políticas laborales es el Ministerio del Trabajo cuyo lema es "Alcanzar el buen vivir, impulsando el empleo digno e inclusivo que garantice la estabilidad y armonía en las relaciones laborales" (Ministerio del Trabajo, 2021).

En cumplimiento de sus políticas laborales el Ministerio de Trabajo, a través de las Direcciones Regionales de Trabajo y estas a su vez por intermedio de las Inspectorías de Trabajo, impulsan la gestión en beneficio de todo el sector laboral es decir empleadores, trabajadores e incluso del propio Estado, a fin de promover el empleo digno y la estabilidad laboral sin embargo hay que mencionar que pese a la evolución 
de la sociedad y a toda la gestión que vienen realizando estas instituciones, a lo largo de los años, las normas laborales no han evolucionado equitativamente, ya que existe ineficacia normativa, así como en el accionar en las autoridades del trabajo, que poca importancia le dan al respeto y observancia del principio de continuidad y seguridad laboral.

De lo citado en líneas anteriores se desprende que la estabilidad laboral es una garantía inherente al trabajador como producto de una relación laboral plenamente establecida. La persistencia y estabilidad del principio de prolongación determinado en apoyo del trabajador, es la razón de ser de la seguridad laboral, y el respeto y cumplimiento a esta garantía significa para el trabajador seguridad económica, desenvolvimiento y habilidad en su labor, una vida tranquila y la satisfacción personal del trabajador y su familia, lo que se traduce como dignidad humana.

Ahora bien, esta estabilidad laboral se ve vulnerada ante la terminación de la dependencia laboral del trabajador, por parte del empleador de una forma ilegal y arbitraria, la cual en el ámbito jurídico lo conocemos como despido intempestivo, cabe indicar, que el despido intempestivo, no está determinado dentro de las causales de culminación de la relación laboral establecida en el artículo 169 del Código de Trabajo, consideradas como causas legales por las cuales se terminar la relación laboral, terminando todo vínculo entre empleador y trabajador (Analuiza, 2018, p. 107).

De lo citado podemos colegir que en el Código de Trabajo no encontramos el concepto o definición del despido intempestivo y por la obvia razón, de que al considerarse como un hecho ilegal el legislador decidió no incorporar este contenido dentro del cuerpo legal laboral, por lo que hemos acudido a la doctrina a fin de inteligenciarnos sobre la definición que varios autores le dan al despido intempestivo, y todos coinciden en que es una forma ilegal, arbitraria e injustificada de dar por concluida la relación laboral por parte del empleador, quebrantando así uno de los principios fundamentales del derecho al trabajo como es la estabilidad laboral y continuidad laboral.

Es importante precisar que, pese a que el despido intempestivo como tal no está contemplado en el Código del Trabajo Ecuatoriano, en el artículo 188 del mencionado cuerpo legal, si se establece la indemnización por despido intempestivo siendo esta 
la parte medular en donde se centra el conflicto laboral y en la mayoría de los casos los empleadores niegan el despido intempestivo para evadir la responsabilidad económica dejando al trabajador en completo estado de vulnerabilidad, momento en el cual el trabajador requiere de forma urgente la protección del Estado, a través de sus diferentes instituciones y funcionarios, en este caso de los Inspectores de Trabajo. En ese contexto, para Gavilánez Criollo (2018):

La estabilidad laboral que es la principal expresión del principio de continuidad, se traduce enfáticamente en la prohibición de los despidos arbitrarios y en caso de ser violado este derecho solo se repararía absolutamente con la restitución del empleo y no con una indemnización, esto es lo que busca la estabilidad propia o absoluta y que se debería aplicar en nuestro País para todos los trabajadores. (p.18)

Las inspectorías del Trabajo tienen un rol fundamental en los casos de los despidos intempestivos, aunque sus funciones se ven limitadas por cuestiones presupuestarias o falta de apoyo administrativo y recursos humanos, sin embargo, es evidente la necesidad de priorizar y mejorar la atención a los sujetos que forman parte del sector laboral en especial a los trabajadores en los casos de despidos intempestivos que han sido negados por parte de los empleadores.

Bajos estas premisas se planteó como objetivo general, analizar jurídicamente la verificación urgente de despidos intempestivos por parte de los Inspectores de Trabajo en Ecuador.

\section{METODOLOGÍA}

En esta investigación, documental se planteó como objetivo general, analizar jurídicamente la verificación urgente de despidos intempestivos por parte de los Inspectores de Trabajo en Ecuador. Se basó en la revisión metódica, rigurosa y profunda de material documental de cualquier clase, con un diseño bibliográfico. (Palella Stracuzzi y Martins Pestana, 2012), a partir de un cúmulo de fuentes documentales, resulta de corte exploratorio a partir de la búsqueda e identificación de la legislación ecuatoriana. Desde el paradigma cuantitativo, se manejó para este tipo de estudio, documentos, textos legales, investigaciones científicas, sobre el tema tratado. Se empleó la técnica de la encuesta, a fin de obtener los resultados del 
proceso metodológico se realizaron encuestas a los abogados en libre ejercicio en materia laboral, tomando como población el registro del Foro de Abogados de Santo Domingo de los Tsáchilas, donde se determinó aleatoriamente una muestra de 65 abogados. Para la aplicación de esta encuesta, se utilizó Google Forms, así como el formato de la escala de Likert.

\section{RESULTADOS}

A continuación, se presentan los resultados obtenidos de la encuesta aplicada a los abogados de libre ejercicio de Santo Domingo de los Tsáchilas.

Interrogantes:

1) ¿Con qué frecuencia, patrocina causas de despido intempestivo?

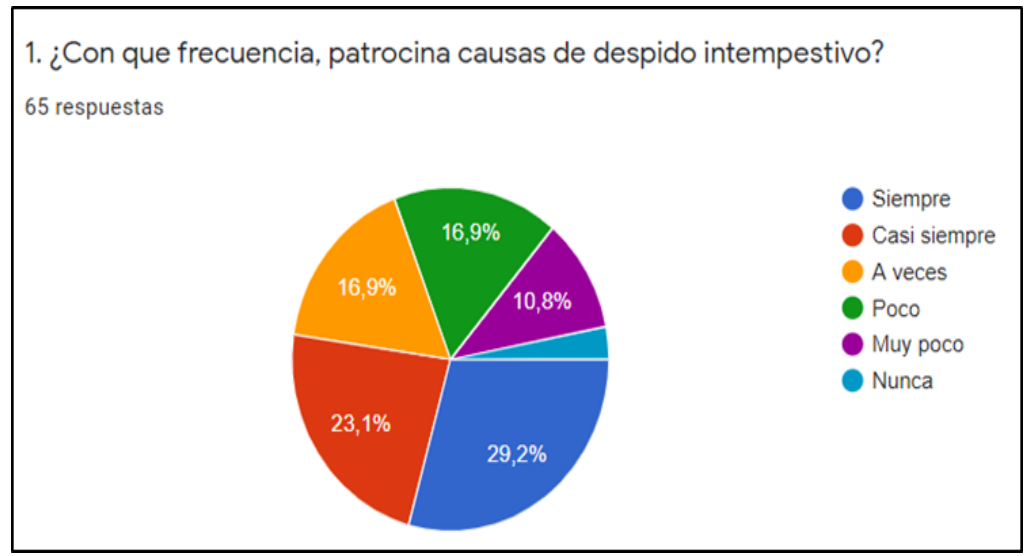

Gráfico 1. Causas de despido intempestivo.

Fuente: Los autores.

De los resultados obtenidos en la primera pregunta se desprende que la mayoría de abogados encuestados, patrocinan causas por despidos intempestivos lo cual significa que existe un número considerable que llegan a sed judicial por cuanto no se resolvió o llegó a una conciliación entre el empleador y trabajador en sed administrativa. 
2) ¿Considera que el despido intempestivo deja en estado de indefensión al trabajador?

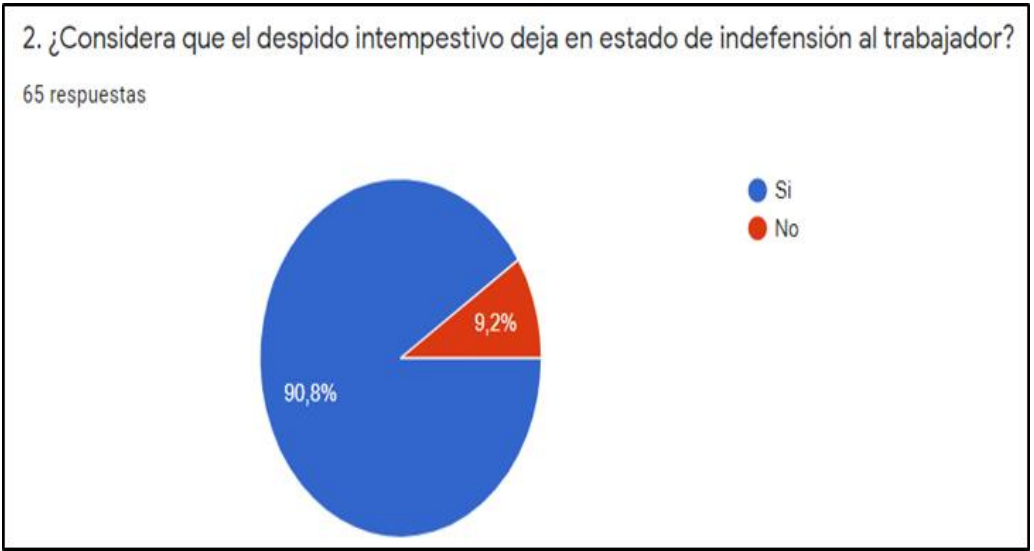

Gráfico 2. Despido intempestivo.

Fuente: Los autores.

Los resultados obtenidos en la segunda pregunta determinan que el criterio de los abogados encuestados en su mayoría considera que, si deja en indefensión, lo cual no permite que el trabajador al menos cobre la indemnización por despido intempestivo y que deberá accionar la vía judicial para acceder a dicho beneficio.

3) ¿Está de acuerdo en que el trabajador tiene los medios de prueba suficientes para demostrar en un proceso judicial que fue despedido intempestivamente?

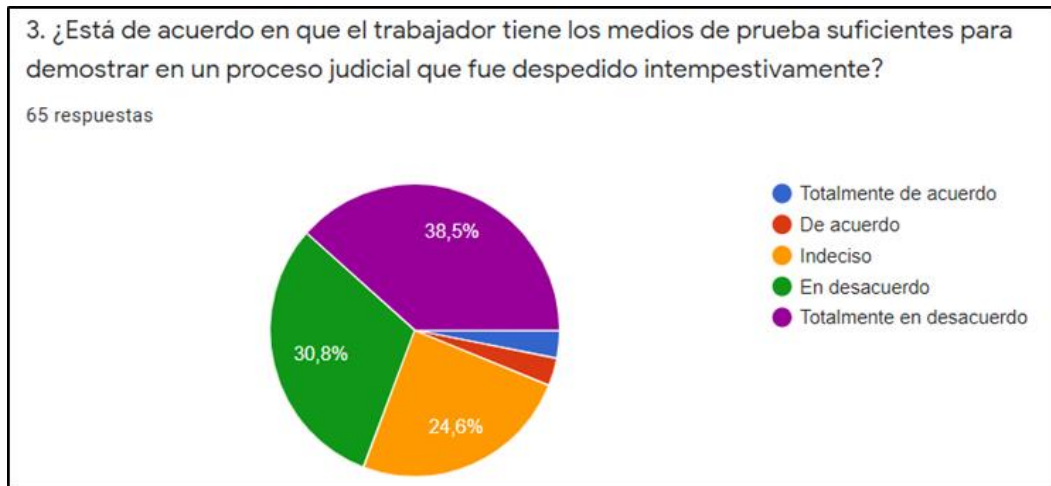

Gráfico 3. Medios de prueba.

Fuente: Los autores. 
En la tercera pregunta los encuestados en su mayoría han expresado su desacuerdo por cuanto en base a sus experiencias en el libre ejercicio son conscientes que al trabajador le resulta muy dificultoso contar con un medio de prueba que demuestre el día, hora, lugar y circunstancias del despido intempestivo.

4) ¿Conoce si los inspectores del trabajo se Santo Domingo realizan visitas o inspecciones con carácter urgente?

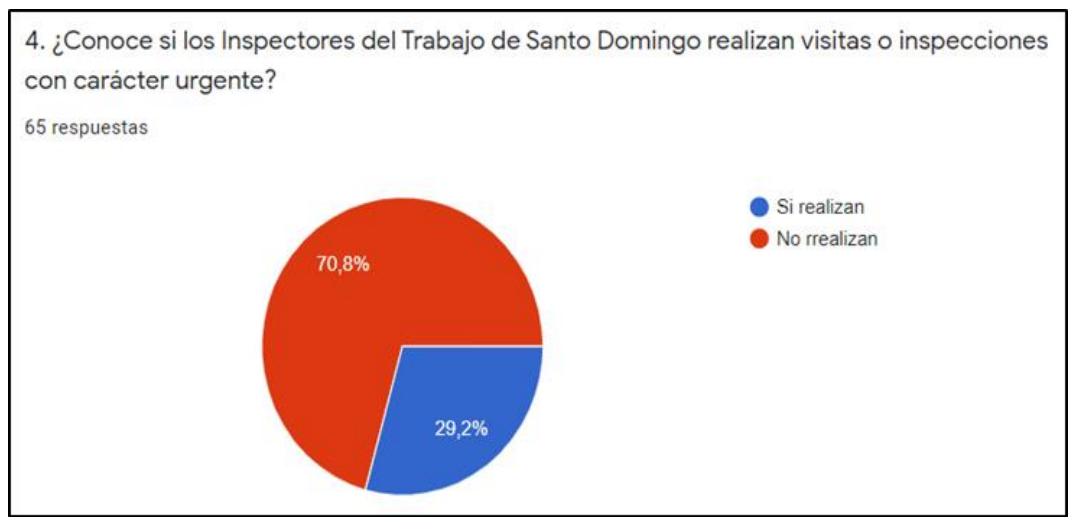

Gráfico 4. Inspecciones

Fuente: Los autores.

En la cuarta pregunta se les planteó dos opciones a los encuestados y la mayoría respondieron que no, lo cual tiene coherencia con los resultados anteriores puesto que si se realizan estas inspecciones o verificaciones con carácter de urgente por parte de los Inspectores del Trabajo de forma inmediata y eficaz se tendría constancia y prueba de los despidos intempestivos lo cual sería una forma d presionar al empleador a que no se termine la relación laboral de forma unilateral e ilegal y consecuentemente el índice de despidos intempestivos sería menor. 
5) ¿Considera que la atención que brindan los inspectores del trabajo de Santo Domingo a los trabajadores es oportuna y eficaz?

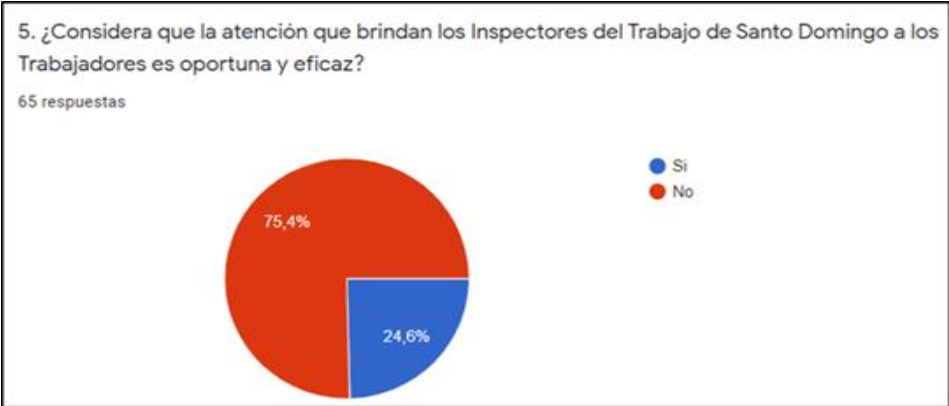

Gráfico 5. Atención al Cliente.

Fuente: Los autores.

En la quinta pregunta las tres cuartas partes de los abogados encuestados en base a sus experiencias en el libre ejercicio, dejan en evidencia que la atención que brindan los inspectores del Trabajo de Santo Domingo no es oportuna y eficaz, frente a una minoría que manifestó lo contrario.

6) ¿Está de acuerdo en si una verificación urgente de los inspectores del trabajo de Santo Domingo ayudaría al trabajar a contar con medios probatorios para demostrar un despido intempestivo?

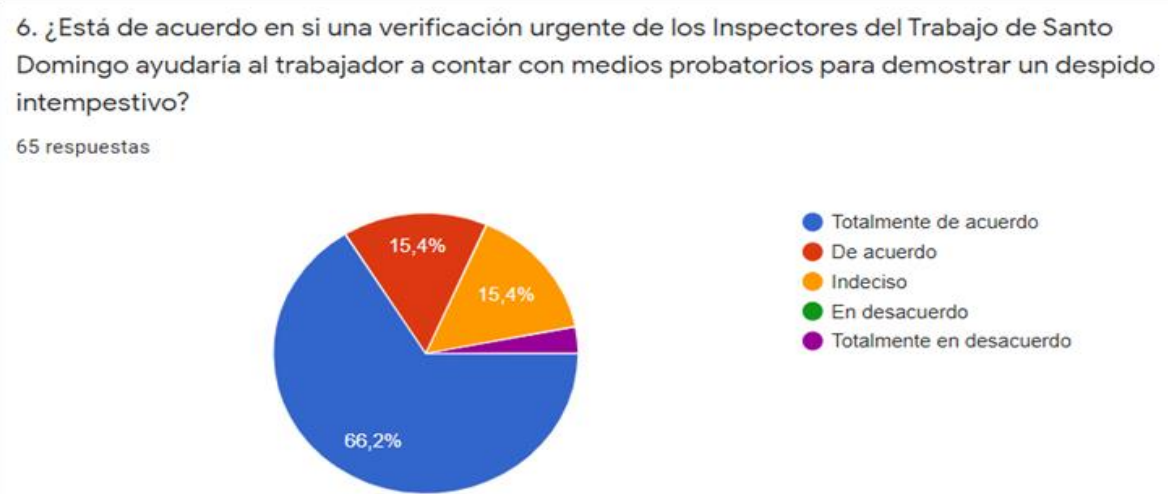

Gráfico 6. Verificación urgente.

Fuente: Los autores. 
Con los resultados obtenidos en la sexta pregunta se refleja un criterio predominante por parte de los abogados encuestados ya que la mayoría están entre totalmente de acuerdo y de acuerdo, frente a un mínimo porcentaje de indecisos, lo cual significa que la mayoría consideran que efectivamente las verificaciones urgentes por parte de los Inspectores del Trabajo coadyuvan al trabajador a la obtención de medios probatorios.

7) A nivel probatorio, ¿considera que el juez de trabajo valora correctamente las pruebas en las causas de despido intempestivo?

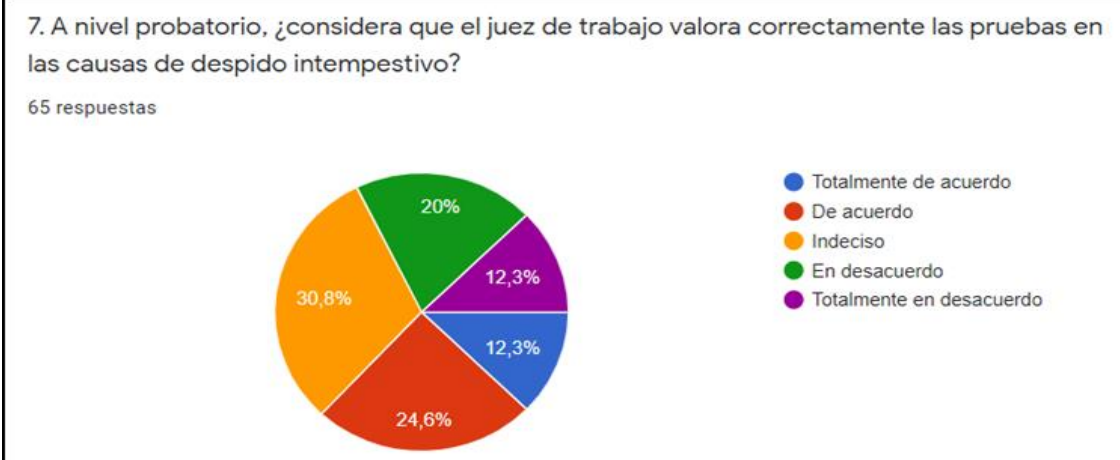

Gráfica 7. Valoración de las pruebas

Fuente: Los autores.

En la séptima pregunta se determina que existen respuestas divididas entre indecisos y que si están de acuerdo y totalmente de acuerdo con la valoración de las pruebas por parte del juez laboral, lo cual se traduce a que definitivamente el trabajador no tiene medios de pruebas necesarios y eficaces para que se le declare el despido intempestivo ya que el juez se basa estrictamente a los medios de prueba para aceptar o negar la pretensión y al no existir medios de pruebas que demuestren el trabajador no puede cobrar su indemnización establecida en el Art. 188 del Código del Trabajo. 


\section{DISCUSIÓN}

En el presente estudio, aplicadas que han sido las técnicas de encuesta y entrevista, obteniendo resultados de carácter investigativo y científico, evidenciando la falta de agilidad en el actuar por parte de los Inspectores del Trabajo de Santo Domingo en relación a prestar un auxilio inmediato al trabajador, a fin de precautelar su estabilidad y continuidad laboral. No obstante, el objeto del presente análisis jurídico se enmarca en la repercusión que tienen verificaciones urgentes ante los despidos intempestivos si los Inspectores del Trabajo lo aplicaran bajo las atribuciones que les confiere el Art. 5 y 542 numeral 5 del Código del Trabajo, lo que significa el compromiso que tiene la jurisdicción administrativa de prestar oportuna protección a los derechos de los trabajadores.

Bajo este lineamiento y en la práctica jurídica, las verificaciones urgentes ante los despidos intempestivos por parte de los Inspectores del Trabajo permitirán dotar al trabajador de mecanismos necesarios a fin de que cuenten con medios de pruebas eficaces que coadyuben a demostrar un despido ilegal, arbitrario y unilateral de la cual fueron objeto por parte del empleador.

Una vez que se realizó la investigación de campo, en este caso de las entrevistas realizadas, se determinó un criterio unánime en relación a la importancia de la función que cumple un Inspector de Trabajo en el ordenamiento jurídico ecuatoriano ya que los entrevistados consideran que la autoridad administrativa tiene como fin velar por las buenas relaciones entre el empleador y trabajador en función a sus atribuciones otorgadas en el Art. 545 del Código del Trabajo.

Uno de los resultados relevantes de analizar es que, los entrevistados coinciden en que en sede judicial la mayoría de las causas, son rechazadas sin mandar a pagar una indemnización por despido intempestivo, por falta de pruebas, ya que al ser considerado el despido intempestivo un hecho cierto el trabajador debe probar día, hora, lugar y circunstancias del despido y que a falta de pruebas, es lógico que el juez rechace la pretensión ya que los jueces resuelven en base a las pruebas que se practiquen en audiencia siendo el trabajador quien tiene que cargar con la prueba que demuestre sus aseveraciones, tal cual lo refiere el artículo 169 de COGEP ley 
supletoria de la normativa laboral, del cual se desprende que es el trabajador quien tiene la carga de la prueba.

En relación a la aplicación de las encuestas los resultados determinaron que efectivamente el despido intempestivo deja en estado de indefensión al trabajador y que por su condición le resulta imposible demostrar el despido, pero sobre todo los encuestados manifestaron que la atención brindada por parte de los Inspectores del Trabajo es deficiente por consiguiente consideran que las verificaciones urgentes es un mecanismo de gran importancia que ayuda al trabajador a que cuente con los medios se prueba eficaces y eficientes para demostrar que efectivamente fue despedido intempestivamente.

\section{CONCLUSIONES}

El Estado a través de sus diferentes Organismos e Instituciones tiene la obligación de proteger y a la vez hacer efectivos los derechos de los trabajadores por intermedio de las autoridades administrativas laborales quienes están obligadas a adoptar todos los medios adecuados y necesarios a fin de velar y custodiar los derechos de la parte considerada vulnerable en la relación laboral como es el trabajador.

La presente investigación corrobora de que, sí es factible y viable que, ante un despido intempestivo, considerado una violación al derecho de estabilidad y continuidad laboral del trabajador, el Inspector del Trabajo debe acudir a realizar una verificación urgente para constar los hechos y levantar un acta dando fe de lo que ha verificado y que dicha acta pueda ser utilizada, como un medio de prueba eficaz a favor del trabajador.

Se ha evidenciado la necesidad por la que atraviesa la Inspectoría del Trabajo de Santo Domingo ya que como institución se ve limitada no solo en recursos económicos sino, en recursos humanos y al existir una gran demanda de casos laborales dichos recursos son insuficientes para prestar un pronto y eficaz auxilio al trabajador.

\section{FINANCIAMIENTO}

No monetario. 


\section{AGRADECIMIENTO}

A la Universidad Regional Autónoma de los Andes, Santo Domingo, por motivar el desarrollo de la Investigación.

\section{REFERENCIAS CONSULTADAS}

Asamblea Nacional (2015) Código Orgánico General de Procesos. [General Organic Process Code]. Registro Oficial № 506. Recuperado de: https://n9.cl/z8haz

Asamblea Nacional Constituyente de la República del Ecuador, (2008). Constitución de la República del Ecuador. [Constitution of the Republic of Ecuador]. Montecristi. Registro Oficial 449 de 20-oct-2008. Recuperado de https://n9.cl/sia

Congreso Nacional (2005) Código de trabajo. [Registro Oficial Suplemento 167. 6 Fecha: 16-dic-2005. Recuperado de: https://n9.cl/ropxu

Chiriboga Izquieta, H., Jiménez, E., y Toscanini Sequeira, P. (2018). El derecho laboral como herramienta política, una mirada histórica. [ Labor law as a political tool, a historical look]. Revista Universidad y Sociedad, 10(1), 226-231. Recuperado de: https://n9.cl/rj74l

Gavilánez Criollo, A. (2018) El contrato de prestación de servicios ocasionales y el principio de estabilidad laboral en el sector público. [ The contract for the provision of occasional services and the principle of job stability in the public sector]. Tesis de pregrado. Universidad Técnica de Ambato. Recuperado de: https://n9.cl/spxso

González Mendoza, O. (2014) El Despido como terminación de la relación laboral en el Ecuador en sus dos proyecciones tempestivo e intempestivo. [Dismissal as termination of the employment relationship in Ecuador in its two projections tempestivo and intempestivo]. Tesis de pregrado. Universidad Laica Vicente Rocafuerte de Guayaquil. Ecuador. Recuperado de: https://n9.cl/25rs9

Masabanda Analuiza, G. (2018) Instrumentos Jurídicos aplicables en la Contratación Individual de Trabajo. [Legal Instruments Applicable to Individual Labor Contracting]. Ambato, Ecuador: Editorial PÍO XII. Recuperado de: https://n9.cl/xrc4s

Ministerio del Trabajo. (2021). Ministerio del Trabajo[ ] El Ministerio - Valores / Misión/ Visión. Recuperado de: https://n9.cl/1a8yu 
Palella Stracuzzi, Santa y Martins Pestana, Feliberto. (2012). Metodología de la investigación cuantitativa. [Quantitative research methodology] Fondo editorial de la Universidad Pedagógica Libertador. Caracas, Venezuela.

Patlán Pérez, J. (2016). Derechos laborales: una mirada al derecho a la calidad de vida en el trabajo. [Labour rights: a look at the right to quality of life at work]. CIENCIA ergo-sum, 23(2),121-133. Recuperado de: https://n9.cl/v9k9 